\title{
The star formation rates of active galactic nuclei host galaxies.
}

\author{
Sara L. Ellison ${ }^{1}$, Hossen Teimoorinia ${ }^{1}$, David J. Rosario ${ }^{2}$, J. Trevor Mendel $^{2}$ \\ ${ }^{1}$ Department of Physics $\mathcal{E}$ Astronomy, University of Victoria, Finnerty Road, Victoria, British Columbia, V8P 1A1, Canada. \\ ${ }^{2}$ Max-Planck-Institut fur Extraterrestrische Physik, Giessenbachstrasse, D-85748 Garching, Germany.
}

14 January 2016

\begin{abstract}
Using artificial neural network (ANN) predictions of total infra-red luminosities $\left(\mathrm{L}_{\mathrm{IR}}\right)$, we compare the host galaxy star formation rates (SFRs) of $\sim 21,000$ optically selected active galactic nuclei (AGN), 466 low excitation radio galaxies (LERGs) and 721 mid-IR selected AGN. SFR offsets ( $\triangle$ SFR) relative to a sample of star-forming 'main sequence' galaxies (matched in $\mathrm{M}_{\star}, z$ and local environment) are computed for the AGN hosts. Optically selected AGN exhibit a wide range of $\triangle \mathrm{SFR}$, with a distribution skewed to low SFRs and a median $\Delta$ SFR $=-0.06$ dex. The LERGs have SFRs that are shifted to even lower values with a median $\triangle \mathrm{SFR}=-0.5 \mathrm{dex}$. In contrast, mid-IR selected AGN have, on average, SFRs enhanced by a factor $\sim 1.5$. We interpret the different distributions of $\Delta$ SFR amongst the different AGN classes in the context of the relative contribution of triggering by galaxy mergers. Whereas the LERGs are predominantly fuelled through low accretion rate secular processes which are not accompanied by enhancements in SFR, mergers, which can simultaneously boost SFRs, most frequently lead to powerful, obscured AGN.
\end{abstract}

Key words: galaxies: active, galaxies: Seyfert, galaxies:interactions

\section{INTRODUCTION}

Nuclear activity is prevalent in star forming galaxies, indicating that a ready supply of matter for the production of stars is linked to the fuelling of active galactic nuclei (AGN). However, the level of star formation in AGN hosts, relative to galaxies that do not exhibit signs of nuclear activity, is contentious. Studies of AGN host galaxies have variously shown that they exhibit elevated (Silverman et al. 2009; Santini et al. 2012; Juneau et al. 2013; Rosario et al. 2015; Magliocchetti et al. 2016), normal (Hatziminaoglou et al. 2010; Harrison et al. 2012; Rosario et al. 2013; Stanley et al. 2015; Xu et al. 2015; Lanzuisi et al. 2015) or suppressed (Salim et al. 2007; Mullaney et al. 2012; Hardcastle et al. 2013; Gurkan et al. 2015; Shimizu et al. 2015; Leslie et al. 2016) levels of star formation, either through 'direct' measures of the star formation rate, SFR, or indirectly through galaxy colour.

At least part of the apparent tension in the literature may be associated with observational methods and biases in the data. For example, since star formation rate is known to correlate closely with stellar mass, and AGN tend be more prevalent in higher mass galaxies, a simple comparison of AGN and non-AGN will be biased towards high SFRs in the former. Comparing SFRs at fixed $M_{\star}$ is therefore essential (e.g. Shimizu et al. 2015; Leslie et al. 2016). Some disagreement between studies may also arise due to the need to either stack or average the data in small samples (Mullaney et al. 2015). Furthermore, the nature of star formation in AGN may evolve as a function of redshift (Magliocchetti et al. 2015). A more subtle point is that AGN may be selected over a broad range of luminosities and via a number of diagnostics, spanning the electro- magnetic spectrum from the X-ray and optical to the mid-infrared (IR) and radio (e.g. see Alexander \& Hickox 2012 for a review). AGN selected at different wavelengths can exhibit very different properties in their accretion rates, environments and host galaxy properties (e.g. Kauffmann et al. 2003; Tasse et al. 2008; Hickox et al. 2009; Smolcic 2009; Best \& Heckman 2012). The different techniques used in studies of different AGN samples makes it difficult to fairly compare the SFRs throughout the AGN family. A more complete understanding of the SFRs in AGN therefore requires both large, sensitive samples for which the distribution of SFRs can be fully parametrized, and a homogeneous comparison between multi-wavelength selected AGN.

One of the fundamental challenges in the pursuit of a homogeneous, multi-wavelength assessment of star formation in AGN is the lack of large samples with uniformly and robustly measured SFRs. Since the AGN itself 'contaminates' the optical recombination lines that are commonly used to calibrate SFRs, these studies have had to adopt alternative methods to determine the SFRs. Two common approaches have emerged in recent years. The first method applies the anti-correlation of $\mathrm{D}_{4000}$ and specific SFR measured in star-forming galaxies (e.g. Brinchmann et al. 2004) and assumes that the same anti-correlation applies in AGN. The advantage of the $\mathrm{D}_{4000}$ SFR calibration is that it is readily applied to galaxies with optical spectroscopy, and SFRs can hence be determined for large numbers of local AGN hosts, such as those in the SDSS. The main disadvantage of the $\mathrm{D}_{4000}$ technique is that its uncertainties are relatively large (Rosario et al. 2016).

More robust SFRs for local AGN may be determined from far IR luminosities which are generally considered to be less contam- 
inated by AGN than other tracers (e.g. Netzer et al. 2007; Buat et al. 2010; Hatziminaoglou et al. 2010; Mullaney et al. 2011). However, the use of IR SFR diagnostics to widely characterize the local galaxy population has so far been relatively limited. Until recently, the main resource for large area studies of galactic IR luminosities were the all sky surveys performed by the Infrared Astronomical Satellite (IRAS) (Neugebauer et al. 1984) and AKARI (Murakami et al. 2007). However, these surveys suffer from poor angular resolution and shallow sensitivity, leading to problems in confusion and detection of only the highest SFR galaxies. The Herschel Space Observatory (hereafter, simply Herschel, Pilbratt et al. 2010), has recently provided a significant step forward in terms of both angular resolution and sensitivity. Several large surveys have been performed with Herschel, such as the Herschel Multi-Tiered Extragalactic Survey (HerMES, Oliver et al. 2012) and the Herschel Stripe 82 survey (Viero et al. 2014). However, even the largest of the Herschel extra-galactic surveys, the Herschel Astrophysical Terahertz Large Area Survey (H-ATLAS, Eales et al. 2010), covered only $\sim 550 \mathrm{deg}^{2}$, which is much smaller than the areas covered by optical spectroscopic surveys such as the SDSS. Therefore, a significant challenge in studying the SFRs of AGN has been assembling samples that are both large, and for which robust (e.g. IR) SFRs are available. Large samples are vital for the measurement of SFR distributions, rather than binned averages which can be dominated by outliers (Mullaney et al. 2015). In Ellison et al. (2016) we presented a catalog of $\sim 330,000$ infra-red luminosities $\left(\mathrm{L}_{\mathrm{IR}}\right)$ for galaxies in the SDSS, derived by artificial neural network (ANN) techniques. In the current work, we use the $\mathrm{L}_{\mathrm{IR}}$ catalog of Ellison et al. (2016) to explore the SFRs of AGN in the local Universe.

\section{METHODOLOGY}

Full details of the $\mathrm{L}_{\mathrm{IR}}$ determinations used in this work are provided in Ellison et al. (2016); only a brief summary is presented here. The Herschel Stripe 82 Survey (Viero et al. 2014) covers a total of $79 \mathrm{deg}^{2}$ in an equatorial strip of the SDSS footprint. Rosario et al. (2016) cross-matched the $250 \mu$ m detected galaxies from $\mathrm{Her}$ schel with the SDSS DR7 Main Galaxy Sample whose extinction corrected Petrosian r-band magnitudes are brighter than 17.77 and whose redshifts are in the range $0.04<z<0.15$, using a positional matching tolerance of 5 arcsecs. The Herschel-SDSS matched catalog contains 3319 galaxies, which were further cross-matched with the Wide Field IR Sky Explorer (WISE, Wright et al. 2010) catalog to yield photometry spanning the mid- to far-IR. Infra-red luminosities were derived by Rosario et al. (2016) by fitting the midand far-IR photometry with the spectral energy distribution templates of Dale \& Helou (2002). AGN are expected to contribute negligibly to the $\mathrm{L}_{\mathrm{IR}}$ of this sample (see Rosario et al. 2016 for more details).

The $\mathrm{L}_{\mathrm{IR}}$ is estimated from an artificial neural network that uses 1136 Herschel detected galaxies with 23 physical parameters, such as stellar masses (Mendel et al. 2014), emission line strengths and photometry (Simard et al. 2011), measured from SDSS data. Based on the popular conversion presented by Kennicutt (1998), the $\mathrm{L}_{\mathrm{IR}}$ may be converted to star formation rates (for a Chabrier initial mass function) using

$\log L_{I R}(\operatorname{erg} / s)=\log S F R\left(M_{\odot} / y r\right)+43.591$.

The SFRs that result from the ANN predictions were shown by Ellison et al. (2016) to be in excellent agreement with those from the SDSS for star-forming galaxies. In this paper, we additionally utilize the $\mathrm{L}_{\mathrm{IR}}$ values of the Ellison et al. (2016) catalog to determine SFRs for AGN host galaxies; indeed, $\mathrm{L}_{\mathrm{IR}}$ SFRs are used throughout this paper, for both star-forming and AGN dominated galaxies, unless otherwise stated. Throughout this paper we require $\sigma_{\text {ANN }}$ (the uncertainty on the ANN predicted $\mathrm{L}_{\mathrm{IR}}$ ) to be less than 0.1 dex $\left(247,137\right.$ galaxeis) and convert $\mathrm{L}_{\mathrm{IR}}$ to SFR using equation 1

In order to compare the SFRs of AGN to those of star-forming (non-AGN) galaxies, we define the SFR offset ( $\triangle \mathrm{SFR}$ ) of a given galaxy $\left(\mathrm{SFR}_{\mathrm{gal}}\right.$ ) relative to a set of comparison star forming (as defined by Kauffmann et al. 2003 and with $\mathrm{S} / \mathrm{N}>3$ ) galaxies that are matched in stellar mass, redshift and local galaxy density (environment), also selected from the SDSS. Local density is defined as $\Sigma_{5}=\frac{5}{\pi d_{5}^{2}}$, where $d_{5}$ is the projected distance in Mpc to the $5^{\text {th }}$ nearest neighbour within $\pm 1000 \mathrm{~km} \mathrm{~s}^{-1}$. Normalized densities, $\delta_{5}$, are computed relative to the median $\Sigma_{5}$ within a redshift slice \pm 0.01 . The baseline tolerance used for matching is $0.1 \mathrm{dex}$ in $\mathrm{M}_{\star}, 0.005$ in $z$ and 0.1 dex in $\delta_{5}$. We require at least 5 comparison galaxies in the matched sample; if this is not achieved then the mass, redshift and local density tolerances are grown in further increments of 0.1 dex, 0.005 and 0.1 dex respectively, until the minimum size criterion of 5 is met. The SFR of the comparison star forming sample $\left(\mathrm{SFR}_{\text {comp }}\right)$ is taken as the median of the matched control sample. The SFR offset is then defined as:

$\triangle S F R=\log S F R_{\mathrm{gal}}-\log S F R_{\text {comp }}$.

\section{RESULTS}

\subsection{Optically selected AGN}

Baldwin, Phillips \& Terlevich (1981) pioneered the use of emission line ratios to distinguish galaxies dominated by various photoionizing processes. Diagnostics separating star-forming from AGN dominated galaxies are now commonly referred to as 'BPT' diagrams, in reference to these authors. In this paper, we make use of three of the most commonly used diagnostics which incorporate the ratios of $[\mathrm{NII}] / \mathrm{H} \alpha$ and $[\mathrm{OIII}] / \mathrm{H} \beta$, by Kewley et al. (2001), Kauffmann et al. (2003) and Stasinska et al. (2006), hereafter K01, K03 and S06 respectively. S06 identifies galaxies with even a small AGN contribution. K03 is slightly more relaxed in its selection of AGN but still includes galaxies that are composites of star formation and AGN. AGN identified by K01 are those dominated by the AGN.

We begin our analysis with a single definition of 'AGN', adopting K03 as our fiducial classification (although our results are qualitatively similar with any of the 3 diagnostics), with a $\mathrm{S} / \mathrm{N}>5$ requirement to minimize the contribution by Low Ionization $\mathrm{Nu}-$ clear Emission line Regions (LINERs; see Leslie et al. 2016 for an assessment of SFR offsets in the LINER population). These selection criteria yield 20,926 AGN with robust $\left(\sigma_{\mathrm{ANN}}<0.1 \mathrm{dex}\right) \mathrm{L}_{\mathrm{IR}}$ predictions. Fig. 1 shows the location of the main sequence of star forming galaxies as filled blue contours. The SFRs of AGN derived from the ANN $\mathrm{L}_{\mathrm{IR}}$ are shown in red contours. For comparison, we also show the aperture corrected AGN SFRs derived from the SDSS spectra via the $\mathrm{D}_{4000}$ method.

As found by previous authors using the $\mathrm{D}_{4000}$ method to measure SFRs (e.g. Salim et al. 2007; Shimizu et al. 2015; Leslie et al. 2016), the optically selected AGN clearly have $\mathrm{L}_{\mathrm{IR}}$ SFRs that lie below the main sequence, i.e. AGN have low SFRs for their $M_{\star}$, typically by $0.05-0.1 \mathrm{dex}$, with a median $\Delta \mathrm{SFR}=-0.06$. The main 


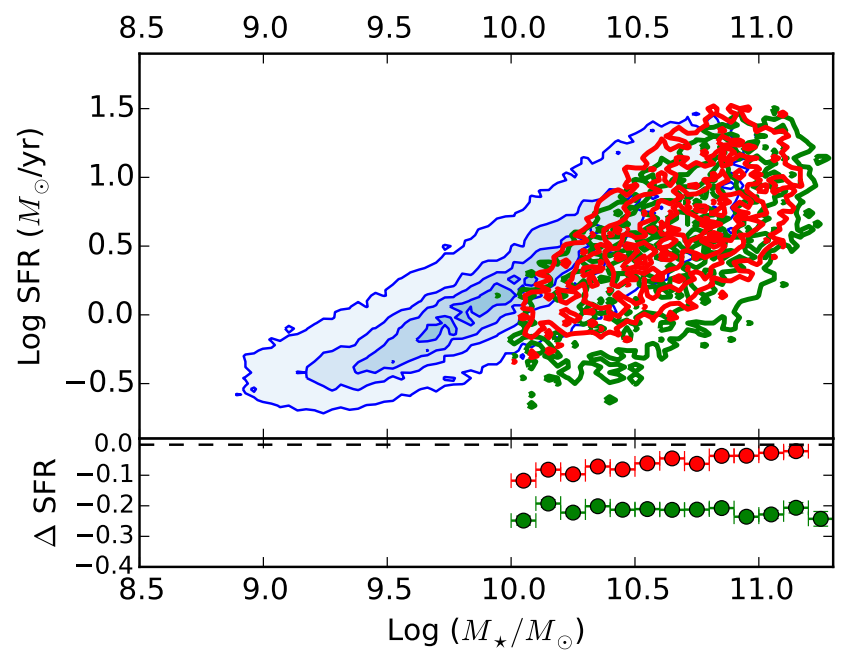

Figure 1. Upper panel: A comparison of star formation rates (from ANN $\mathrm{L}_{\mathrm{IR}}$ estimates) in star forming galaxies (filled blue contours) and AGN (open contours) classified according to Kauffmann et al. (2003). Lower panel: The offset between SFRs in AGN (as measured from $\mathrm{L}_{\mathrm{IR}}$ ) relative to the main sequence. In both panels, red and green colours refer to SFRs derived from ANN $L_{I R}$ predictions and from $\mathrm{D}_{4000}$ measurements, respectively.

sequence offsets measured using $\mathrm{D}_{4000}$ extended to somewhat lower SFRs than the $\mathrm{L}_{\mathrm{IR}}$ derived values. There are at least two possible reasons for this. First, the SFRs derived from the ANN are limited to about $0.3 \mathrm{M}_{\odot} \mathrm{yr}^{-1}$, due to the detection limit in the training set. It is therefore likely that there is a further tail of low SFR AGN that are not included in our sample due to an effective 'detection limit' in the ANN $\mathrm{L}_{\mathrm{IR}}$ predictions (e.g. Leslie et al. 2016). A second possible reason for the more extended green contours is that, as shown by Rosario et al. (2016) and Ellison et al. (2016), the $\mathrm{D}_{4000}$ based SFRs are much more uncertain than those derived from $\mathrm{L}_{\mathrm{IR}}$, so the lower SFRs in the former may be a result of a broader error disribution.

In order to investigate the dependence of $\triangle$ SFR on the relative contribution of the AGN, in Fig. 2 we plot the BPT diagram for AGN colour-coded by $\triangle$ SFR. In order to explore the dependence of $\triangle$ SFR over the maximum range on the BPT diagram, we 'relax' the definition of AGN by adopting the criteria of S06, but also showing the demarcation lines fo K01 and K03. There are 33,300 galaxies that are classified as AGN by the S06 criterion for which we have ANN $L_{\text {IR }}$ predictions and for which $\triangle$ SFR can be determined. To avoid crowding on Fig. 2 we plot a random sample of 5000 AGN. For reference, all three commonly used diagnostics (K01, K03, S06) are shown in Fig. 2 as black lines. Negative values of $\Delta$ SFR (low SFRs) dominate throughout the AGN 'wing'. The exception is very close to the S06 demarcation, where there appear to be fewer galaxies with suppressed SFRs. Despite the general preference for AGN to exhibit low $\triangle$ SFRs, there is a wide variety of SFR enhancements at most locations. Fig. 2 demonstrates that, although there is a trend towards more suppressed SFRs as we move along the AGN branch (see also Leslie et al. 2016), even galaxies that are dominated by AGN can sometimes exhibit strong SFR enhancements (see also the middle panel of Fig. 3). An important caveat to these conclusions is that samples of optically selected AGN may be inherently biased against galaxies with very high SFRs. Trump et

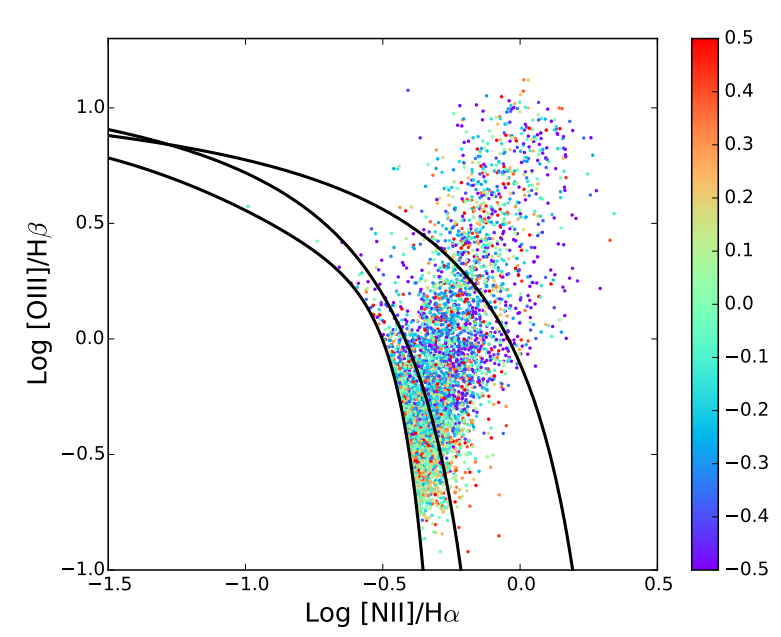

Figure 2. BPT diagram colour coded by $\triangle$ SFR for AGN.The 3 black lines show the AGN demarcations of (from top to bottom), K01, K03 and S06. A random sample of 5000 out of $\sim 33,300$ AGN selected by S06 with $\mathrm{L}_{\mathrm{IR}}$ based SFRs is shown.

al. (2015) have investigated the effect of star formation 'dilution', whereby high SFRs can move even moderately powerful AGN onto the star forming branch of the BPT diagram. The dilution effect is most problematic for low mass galaxies. However, as shown in the lower panel of Fig. 1 the median value of $\Delta$ SFR in optically selected AGN does not depend strongly on $\mathrm{M}_{\star}$, indicating that any bias against the identification of highly star-forming AGN is not driving the tendency towards suppressed SFRs.

For the remainder of this paper, we use the K03 classification as our fiducial definition of 'optical' AGN.

\subsection{AGN selected in the IR and radio}

The physical properties, such as stellar mass distribution, morphological structure and average colours, of AGN host galaxies depend strongly on how the AGN are selected (e.g. Hickox et al. 2009). In this sub-section, we determine SFR offsets from the main sequence for two other samples of AGN, one selected based on radio emission, and the other based on mid-IR colour selection. The novelty of our work is that we can make a direct comparison of SFR offsets for these three populations based on a homogeneous analysis.

The sample of low luminosity radio-selected AGN, specifically the low excitation radio galaxies (LERGs), is taken from the SDSS catalog compiled by Best \& Heckman (2012). We determine the SFRs of the Best \& Heckman LERG catalog from our ANN LIR predictions; there are 466 LERGs for which $\sigma_{\text {ANN }}<0.1$.

The sample of mid-IR selected AGN is identified by crossmatching the WISE All Sky Survey (Wright et al. 2010) with the SDSS and requiring a maximum angular separation of 6 arcsecs. We use the $\mathrm{W} 1$ and $\mathrm{W} 2$ band profile magnitudes, with a minimum $\mathrm{S} / \mathrm{N}$ requirement of 5 and identify AGN as galaxies exceeding W1-W2>0.8 (Stern et al. 2012). 721 of these WISE selected AGN have ANN $\mathrm{L}_{\mathrm{IR}}$ with $\sigma_{\mathrm{ANN}}<0.1$.

In Fig. 3 we show the SFR offsets of the three AGN samples (coloured histograms), as well as the distribution of $\triangle$ SFR amongst star-forming galaxies (black line). By definition, this latter distribution should be centred at $\Delta \mathrm{SFR}=0$, since the star forming galaxies define the main sequence from which offsets are computed; the 


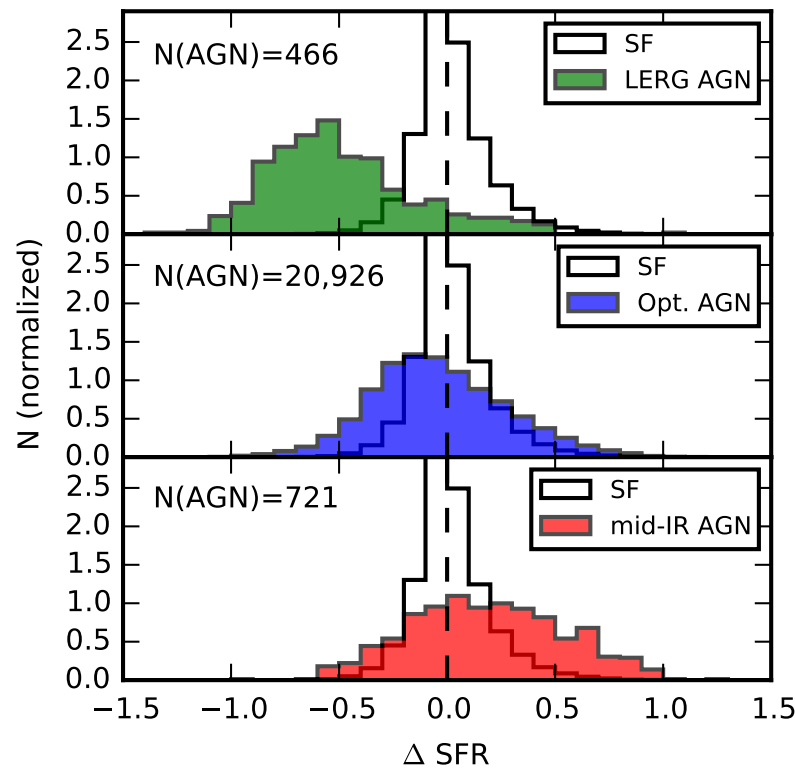

Figure 3. Distributions of $\Delta$ SFR for various classes of AGN. In all panels, the distribution of $\Delta$ SFR in star forming galaxies is shown in black; by definition, this distribution is centred around $\triangle \mathrm{SFR}=0$.

width of the histogram simply shows the scatter in the main sequence. In the upper panel of Fig. 3 we confirm the result of Gurkan et al. (2015), who studied LERGs in the H-ATLAS sample, that LERGs are offset to lower SFRs than the star forming main sequence. The optically selected AGN (K03) are shown in the blue histogram of the middle panel of Fig. 3 By comparing the top and middle panels of Fig. 3 it can be seen that, whilst both the LERGs and the optically selected AGN have a modal $\triangle$ SFR that is negative, the distributions are quite different. On average, the LERGs are shifted to much lower values of $\Delta$ SFR than the optically selected AGN; the median offset is $\triangle \mathrm{SFR}=-0.5$. Whilst 65 per cent of optically selected AGN lie within a factor of two of the main sequence, and can even exhibit enhanced SFRs, the LERGs rarely attain the main sequence SFR for their stellar mass.

In the lower panel of Fig. 3 we show the distribution of SFR offsets for AGN identified in the mid-IR. For the first time, we can now compare the SFR offsets in a homogeneous way for the three AGN populations. In contrast to the optically selected AGN and the LERGs, the median $\Delta$ SFR of mid-IR selected AGN is +0.17 dex, indicating that the typical powerful, dust-obscured AGN has a factor of $\sim 1.5$ excess SFR compared with the main sequence. However, the tail of the $\Delta$ SFR distribution extends up to +1 dex, indicating that some mid-IR selected AGN have SFRs a factor of 10 above their comparison sample. Such elevated SFRs are absent in the LERG sample, and rare amongst the optically selected AGN. In Ellison et al. (2016) we showed that the ANN predictions of $\mathrm{L}_{\mathrm{IR}}$ were accurate even when the SFR was elevated to these levels above the main sequence.

\section{DISCUSSION AND CONCLUSIONS}

What drives the difference between the $\triangle$ SFR distributions of the 3 types of AGN shown in Fig. 3. In particular, why are the SFRs in mid-IR selected AGN enhanced relative to the main sequence, whereas LERGs have SFRs that are almost universally below expectations for their $M_{\star}$ (see also Gurkan et al. 2015)? Hardcastle et al. (2013) posed a similar question after finding that high excitation radio galaxies (HERGs) had enhanced SFRs, in contrast to the low SFRs in LERGs. Hardcastle et al. (2013) proposed this difference was due to the environments in which LERGs an HERGs are typically located (Best \& Heckman 2012). However, since our starforming comparison sample is matched in $\delta_{5}$, this seems unlikely to be the explanation for the different $\Delta$ SFR distributions seen in Fig. 3 .

Our finding that IR, optical and radio-selected AGN form a sequence in $\Delta$ SFR is consistent with an evolutionary picture in which an abundant gas supply triggers enhanced star formation in an obscured AGN phase. As the fuel supply declines, so does the star formation rate and the obscuration. A similar picture has been proposed by Hickox et al. (2009), based on a comparison between the host galaxy and clustering properties of a sample of IR, X-ray and radio selected AGN. Hickox et al. (2009) found that AGN selected in these ways formed a sequence in decreasing star formation and accretion rate, and increasing halo mass. The evolutionary sequence between star formation and obscuration is further supported by the observation that, for samples of X-ray selected AGN, star formation rates are significantly enhanced for the obscured/absorbed AGN, but are normal or suppressed for unobscured AGN (Stevens et al. 2005; Juneau et al. 2013). Moreover, the X-ray absorbed AGN fraction increases as galaxies lie progressively above the star forming main sequence (Juneau et al. 2013).

Galaxy mergers are a natural mechanism to explain such an evolutionary sequence, as first proposed by Sanders et al. (1988), since mergers are known to be capable of triggering both star formation (e.g. Scudder et al. 2012) and AGN activity (e.g. Ellison et al. 2011). In this scenario, the merger remnant of a gas-rich, major merger first undergoes a significant starburst, possibly manifesting as a luminous (or ultra-luminous) IR galaxy and high accretion rate AGN (possibly a quasar). As the starburst fades, the AGN transitions from obscured to unobscured and the galaxy eventually evolves to an early type (e.g. see Fig. 6 in Alexander \& Hickox 2012 for a schematic view of this process).

The dependence of star formation rate enhancement on AGN obscuration during the post-merger phase is supported by the observation that certain classes of AGN are more prevalent amongst ongoing mergers. For example, in close pairs and recent post-mergers, optically selected AGN are over-abundant (relative to the AGN frequency in isolated galaxies) by a factor of 2-3 (Ellison et al. 2013), compared to a factor of 5-20 in mid-IR selected AGN (Satyapal et al. 2014). Mid-IR selected AGN have also been shown to exhibit a high dual black hole fraction (Secrest et al., in preparation), in contrast to optical searches which have a low success rate in detecting binaries (Fu et al. 2011; Muller-Sanchez et. al 2015). The connection between obscuration and merging is also found in the $\mathrm{X}$-ray, where the most absorbed AGN are found most frequently in mergers (Kocevski et al. 2015; Lanzuisi et al. 2015).

In contrast to the consistent link between obscured AGN and mergers, the results for the unobscured population has been more controversial. Whereas an excess of AGN is measured in close pairs of galaxies (Ellison et al. 2011; Silverman et al. 2011), demonstrating that mergers may trigger AGN, the morphological classification of AGN indicates that mergers do not appear to dominate the triggering of unobscured AGN (e.g. Gabor et al. 2009; Kocevski et al. 2012). These results are consistent with the distribution of SFRs in optically selected AGN (Fig 3 , middle panel), in which the major- 
ity of galaxies have normal-to-low SFRs, and are hence unlikely to be linked to mergers. The small number of optical AGN with enhanced SFRs may be those that are merger triggered, a postulate that is confirmed through visual inspection of the SDSS images.

In contrast to optical and mid-IR selected AGN, there is no excess of LERGs in merger samples, indicating that they are fuelled by secular processes, where low levels of accretion (not connected with a starburst) can be maintained from a combination of stellar and external sources (Ellison, Hickox \& Patton 2015). Rather than an 'evolutionary' (i.e. time sequence) scenario, our observations may therefore also be explained by different relative contributions of mergers to the 3 AGN classes. I.e. LERGs have the lowest SFRs because there is no boost from mergers, whereas a higher fraction of IR selected AGN are linked to mergers and have experienced triggered star formation during the interaction. Put another way, mergers may lead to high SFRs and luminous (obscured) AGN, but a distinct, secular pathway is responsible for lower luminosity AGN with normal or low SFRs (e.g. Shao et al. 2010).

The idea that the distribution of $\Delta$ SFR in the different AGN classes is by driven by the relative contributions of mergers is supported by differences in their merger fractions. We compute the merger fraction for each AGN class as the fraction of galaxies classified as a merger in Galaxy Zoo, requiring a merger vote fraction $>$ 0.4 (e.g. Darg et al. 2010). Merger fractions for the different types of AGN are: radio - 1 per cent, optical - 3 per cent, mid-IR - 7 per cent. The higher merger fraction amongst the mid-IR sample is despite a redshift distribution skewed towards slightly higher values than the optical AGN and LERG samples making it potentially harder to identify mergers therein. Although these merger fractions should not be considered as absolute, due to the limitations of visual classification in shallow ground-based images, without removal of projected companions, their relative values are indicative that mergers are most prevalent amongst IR selected AGN, and least common amongst LERGs. A complete assessment of mergers in this sample is beyond the scope of this paper, but we note that the excess of mergers amongst mid-IR selected persists for stricter cuts on merger vote fraction.

In conclusion, our main result is that the SFRs of AGN are critically dependent on how an AGN is selected. IR selected galaxies in the SDSS show a median SFR enhancement of a factor of $\sim$ 1.5 , compared to an under-abundance of star formation in optically selected AGN (by $\sim 25$ per cent) and low luminosity radio-selected AGN (by a factor of 3). We propose that these differences may be explained by a relatively high fraction of mergers amongst IR selected AGN, a lower merger incidence amongst optically selected AGN, and the domination of secular fuelling processes for LERGs, which are hosted primarily by massive galaxies with low SFRs.

\section{REFERENCES}

Alexander, D. M., Hickox, R. C., 2012, NewAR, 56, 93

Baldwin, J. A,; Phillips, M. M., Terlevich, R., 1981, PASP, 93, 5

Best, P. N., Heckman, T. M., 2012, MNRAS, 421, 1569

Brinchmann, J., Charlot, S., White, S. D. M., Tremonti, C., Kauffmann, G., Heckman, T., Brinkmann, J.,2004, MNRAS, 351, 1151

Buat, V., et al., 2010, MNRAS, 409, 1

Dale, D. A., \& Helou, G., 2002, ApJ, 576, 159

Darg, D. W., et al., 2010, MNRAS, 401, 1552

Eales, s., et al., 2010, PASP, 122, 499

Ellison, S. L., Mendel, J. T., Patton, D. R., Scudder, J. M., 2013, MNRAS, 453,3627

Ellison, S. L., Patton, D. R., Hickox, R. C., 2015, MNRAS, 451, L35
Ellison, S. L., Patton, D. R., Mendel, J. T., Scudder, J. M., 2011, MNRAS, 418, 2043

Ellison, S. L., Teimoorinia, H., Rosario, D. J., Mendel, J. T., 2016, MNRAS, 455,370

Fu, H., Myers, A. D., Djorgovski, S. G., Yan, L., 2011, ApJ, 733, 103

Gabor, J. M., et al., 2009, ApJ, 691, 705

Gurkan, G., et al., 2015, MNRAS, 452, 3776

Hardcastle, M. J., et al., 2013, MNRAS, 429, 2407

Harrison, C. M., et al., 2012, ApJ, 760, L15

Hatziminaoglou, E., et al., 2010, A\&A, 518, L33

Hickox, R. C., et al., 2009, ApJ, 696, 891

Juneau, S., 2013, ApJ, 764, 176

Kauffmann, G., et al., 2003, MNRAS, 346, 1055

Kennicutt, R.C. 1998, ARA\&A, 36, 189

Kewley, L. J., Dopita, M. A., Sutherland, R. S., Heisler, C. A., Trevena, J., 2001, ApJ, 556, 121

Kocevski, D., et al. 2012, ApJ, 744, 148

Kocevski, D., et al. 2015, ApJ, 814, 104

Lanzuisi, G., et al., 2015, A\&A, 573, 137

Leslie, S. K., Kewley, L. J., Sanders, D. B., Lee, N., 2016, MNRAS, 455, L82

Magliocchetti, M., Lutz, D., Santini, P., Salvato, M., Popesso, P., Berta, S., Pozzi, F., 2016, MNRAS, 456, 431

Mendel, J. T., Palmer, M. J. D., Simard, L., Ellison, S. L., Patton, D. R., 2014, ApJS, 210, 3

Mullaney, J. R., Alexander, D. M., Goulding, A. D., Hickox, R. C., 2011, MNRAS, 414, 1082

Mullaney, J. R., et al., 2012, ApJ, 419, 95

Mullaney, J. R., et al., 2015, MNRAS, 453, L83

Muller-Sanchez, F., Comerford, J. M., Nevin, R., Barrows, R. S., Cooper, M. C., Greene, J. E., 2015, ApJ, 813, 103

Murakami, H., et al., 2007, PASJ, 59S, 369

Netzer, H., et al., 2007, ApJ, 666, 806

Neugebauer, G., et al., 1984, ApJ, 278, L1

Oliver, S. J., et al., 2012, MNRAS, 424, 1614

Pilbratt, G. L., et al., 2010, A\&A, 518, 1

Rosario, D. J., et al., 2013, A\&A, 560, 72

Rosario, D. J., et al., 2015, A\&A, 573, 85

Rosario, D. J., Mendel, J. T., Ellison, S. L., Lutz, D., Trump, J. R., 2016, MNRAS, in press

Salim, S., et al, 2007, ApJS, 173, 267

Sanders, D. B., Soifer, B. T., Elias, J. H., Madore, B. F., Matthews, K., Neugebauer, G., Scoville, N. Z.., 1988, ApJ, 325, 74

Santini, P., et al., 2012, A\&A, 540, 109

Satyapal, S., Ellison, S. L., McAlpine, W., Hickox, R. C., Patton, D. R., Mendel, J. T., 2014, MNRAS, 441, 1297

Scudder, J. M., Ellison, S. L., Torrey, P., Patton, D. R., Mendel, J. T., 2012, MNRAS, 426, 549

Shao, L., et al., 2010, A\&A, 518, L26

Shimizu, T. T., Mushotzky, R. F., Melendez, M., Koss, M., Rosario, D. J., 2015, MNRAS, 452, 1841

Silverman, J. D., et al., 2009, ApJ, 696, 396

Simard, L., Mendel, J. T., Patton, D. R., Ellison S. L., McConnachie, A. W., 2011, ApJS, 196, 11

Smolcic, V., 2009, ApJ, 699, L43

Stanley, F. et al., 2015, MNRAS, 453, 591

Stasinska, G., Cid Fernandes, R., Mateus, A., Sodre, L., Asari, N. V., 2006, MNRAS, 371, 972

Stern, D., et al., 2012, ApJ, 753, 30

Stevens, J. A., Page, M. J., Ivison, R. J., Carrera, F. J., Mittaz, J. P. D., Smail, Ian, McHardy, I. M., 2005, MNRAS, 360, 610

Trump, J. R., et al., 2015, ApJ, 811, 26

Tasse, C., Best, P. N., Rottgering, H., Le Borgne, D., 2008, A\&A, 490, 893

Viero, M. P., et al., 2014, ApJS, 210, 22

Wright, E. L., et al., 2010, AJ, 140, 1868

Xu, L., Rieke, G. H., Egami, E., Pereira, M. J., Haines, C. P., Smith, G. P., 2015, ApJS, 219, 18 